\title{
Association between toe grasping strength and accelerometer-determined physical activity in middle-aged and older women
}

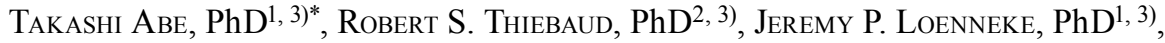 \\ Naotoshi Mitsukawa, $\mathrm{PhD}^{3)}$ \\ 1) Department of Health, Exercise Science, and Recreation Management, Kevser Ermin Applied \\ Physiology Laboratory, School of Applied Sciences, The University of Mississippi: 215 Turner \\ Center, University, MS 38677, USA \\ 2) Department of Kinesiology, Texas Wesleyan University, USA \\ 3) Active Aging Research Center, Toyo Gakuen University, Japan
}

\begin{abstract}
Purpose] To test the hypothesis that toe grasping strength is associated with daily physical activity in older adults. [Subjects] Fifty-seven Japanese women, aged 52-78 years, volunteered. [Methods] Toe grasping and knee extension strength were measured. Physical activity was also measured, using an accelerometer, and the total duration of each level of exercise intensity (light, moderate, and vigorous) and average step counts were calculated. Subjects were separated into two groups on the basis of accelerometer-determined step counts: LOW ( $\mathrm{n}=28,<8000$ steps/day) and HIGH ( $\mathrm{n}=29, \geq 8000$ steps/day). [Results] Body mass index and body composition ( $\%$ fat and fat-free mass) were similar between the two groups. Absolute and relative toe grasping strengths (divided by body weight) were greater in HIGH than in LOW. However, both absolute and relative knee extension strength were similar between the groups. Relative toe grasping and knee extension strength correlated with all 3 intensities of physical activity and average step count. After adjusting for age, the duration of light plus moderate physical activity and average step counts correlated to toe grasping strength but not to knee extension strength. [Conclusion] Our results suggest that toe grasping strength may be associated with the amount of light intensity daily physical activity.

Key words: Accelerometer, Isometric contraction, Foot muscle strength
\end{abstract}

(This article was submitted Jan. 28, 2015, and was accepted Mar. 7, 2015)

\section{INTRODUCTION}

Muscle strength is essential for posture and stability. In a standing posture, the foot is the point of direct contact between the body and the surface below. Therefore, muscle force generated from the toes and ankles may play an important role in maintaining stability. A limited number of studies have reported the association between toe grasping strength and postural control. For example, two studies investigated the age-related change in toe grasping strength in males and females and found that toe grasping strength is related to age and declines faster than handgrip strength with increasing age ${ }^{1,2)}$. In addition, Handa and colleagues ${ }^{1)}$ reported significant correlations between toe grasping strength and static balance with eyes open $(r=0.443, p<0.01)$ as well as tolerance to falling forward $(r=0.620, p<0.01)$. Interestingly, postural sway behavior while standing improved fol-

*Corresponding author. Takashi Abe (E-mail: t12abe@gmail. com)

C2015 The Society of Physical Therapy Science. Published by IPEC Inc. This is an open-access article distributed under the terms of the Creative Commons Attribution Non-Commercial No Derivatives (by-ncnd) License $<$ http://creativecommons.org/licenses/by-nc-nd/3.0/> lowing 8 weeks of toe grasp training (consisting of gathering a towel attached to a weight and passing small bags from one place to another) in older adults although the authors did not measure toe grasping strength ${ }^{3}$.

It is known that balance control may be influenced by daily physical activity ${ }^{4-6}$. Perrin and colleagues ${ }^{4}$ reported that daily physical activity in older adults, even if never practiced earlier in their life, had a positive effect on balance control. Gauchard and colleagues ${ }^{5)}$ investigated the influence of two types of physical exercise (yoga and soft gymnastics vs. jogging, swimming, and cycling) on postural sway balance in older females and found that yoga and soft gymnastics appeared to have the best impact on postural control in simple postural tasks. One difference between these two types of physical exercise is how the foot contacts the ground surface during the exercise. Foot muscles involved in toe grasping may be activated during relatively slow movements of physical activities such as yoga and soft gymnastics. Thus, toe grasping strength may be associated with the duration and intensity of daily physical activity. The purpose of the present study was to test the hypothesis that daily physical activity probably contributes to toe grasping strength in middle-aged and older adults. 


\section{SUBJECTS AND METHODS}

Fifty-seven Japanese women between the ages of 52 and 78 years (mean age, $66.3 \pm 6.8$ years) were recruited through printed advertisement and by word of mouth from the surrounding area of the university campus in Chiba. In this study, female volunteers were chosen because a higher incidence of falls has been reported in Japanese females than in Japanese males ${ }^{7)}$. All subjects were free of overt chronic disease (e.g., diabetes, angina, myocardial infarction, arthritic and neuromuscular disorders, cancer, stroke) as assessed by self-report following a general health examination. Approximately $68 \%$ of subjects performed regular sports activity at least once a week. The main types of sports activities were walking (17 females) and Yoga/Tai Chi (6 females). The study was conducted according to the Declaration of Helsinki and was approved by the Ethics Committee for Human Experiments of Toyo Gakuen University (2012-001). Written informed consent was obtained from all subjects.

Subcutaneous fat thickness (FT) was measured using Bmode ultrasound (Aloka SSD-2000, Tokyo, Japan) at 9 sites, as described previously ${ }^{8)}$. The measurements were taken while the subjects stood quietly with their knees extended and relaxed. A 5-MHz scanning head was placed on the measurement site without depressing the dermal surface. Body density was estimated from FT using an ultrasound-derived prediction equation ${ }^{8)}$. Percent body fat (\%fat) was calculated from body density using Brozek's equation ${ }^{9)}$. Fat-free mass (FFM) was estimated as total body mass minus fat mass. Body mass and standing height were measured to the nearest $0.1 \mathrm{~kg}$ and $0.1 \mathrm{~cm}$, respectively, using a height scale and an electronic weight scale. Body mass index (BMI) was defined as body mass $(\mathrm{kg}) /$ height $^{2}\left(\mathrm{~m}^{2}\right)$.

Toe grasping strength was measured using a toe-grasp dynamometer (T.K.K. 3361, Takei, Tokyo, Japan), as described previously ${ }^{2}$. While barefoot, subjects were instructed to maintain a one-legged upright standing position on the dynamometer, put both hands on the wall in front of them, and hold the dynamometer grasping bar with their toes. The distance between the bar and the heel was adjusted to the foot size of each subject such that the distal phalanges of the great toe and fifth toe and the middle phalanges of the second to fifth toes could be placed on the toe grasping bar. Subjects were allowed to perform one test trial, followed by 3 maximum trials, and the best values for right and left feet were averaged and used for data analysis. Maximal toe grasping strength divided by body weight was calculated for evaluating relative toe grasping strength. Test-retest reliability of toe grasping strength has been reported in a previous study ${ }^{10)}$.

Maximum voluntary isometric strength of the knee extensors was determined using a Biodex System 3 dynamometer (Shirley, New York, USA). Subjects were carefully familiarized with the testing procedures of voluntary force production approximately one week before testing. Each subject was seated on a chair with the hip joint angle positioned at $85^{\circ}$. The center of rotation of the knee joint of the right leg was visually aligned with the axis of the lever arm of the dynamometer, and the ankle of the right leg was firmly attached to the lever arm of the dynamometer with a strap.
After a warm-up consisting of submaximal contractions, the subject was instructed to perform maximal isometric (MVC) knee extension at a knee joint angle of $80^{\circ}$. A knee joint angle of $0^{\circ}$ corresponded to full extension of the knee. If MVC strength for the first two trials varied by $>5 \%$, an additional MVC was performed. The coefficient of variation for this test in our laboratory was 7\%. Knee extension MVC divided by body weight was calculated for evaluating relative knee extension strength.

During orientation, participants were shown how to attach the accelerometer (Lifecorder EX, Suzuken, Nagoya, Japan) on an elastic belt over their left or right hip and were instructed to record their daily physical activity for 30 days, beginning on the following day. If participants did not use the accelerometer for a couple of days during the testing period, they were requested to record their activity for additional days. To achieve $>90 \%$ reliability for estimating the yearly physical activity in older adults, $>30$ consecutive observation days are required ${ }^{11)}$. After the measurements were taken, the recorded activity level data were downloaded to a personal computer. The data were classified into different intensities using 10 activity levels, ranging from 0 to 9 , based on the accelerometer signal. Level 0 (corresponding to $<0.06 \mathrm{G}$ ) denotes immobility, and levels 1 to 9 (corresponding to $\geq 0.06 \mathrm{G}$ ) denote subtle to vigorous movements. In this study, exercise intensity was categorized into one of 3 activity levels on the basis of the accelerometer signal: light physical activity (levels $1-3,<3$ metabolic equivalents $[\mathrm{MET}]$ ), moderate physical activity (levels 4-6, 3-6 MET), and vigorous physical activity (levels 7-9, > 6 MET) according to a previous study ${ }^{12)}$. In addition, the total duration of each level of exercise intensity was calculated. The average daily step count was also determined using the accelerometer.

Results are expressed as mean and standard deviation for all variables. Participants were separated into two groups on the basis of daily step counts measured by an accelerometer: LOW ( $\mathrm{n}=28,<8,000$ steps per day) and HIGH ( $\mathrm{n}=29$, $\geq 8,000$ steps per day). Before comparisons were made, dependent variables were tested for normality of distribution by the Shapiro-Wilk test. The difference between LOW and HIGH groups was tested for significance by using unpaired Student's t-tests, and if any variables were not normally distributed, the Mann-Whitney $U$ test was used. Pearson product correlations were performed to determine the relationships between accelerometer-determined physical activity level and relative maximum strength (divided by body weight) of toe grasping and knee extension. If any variables were not normally distributed, then Spearman's rho correlation was used. Because age was different between the two groups, partial correlations of relative strength with physical activity levels adjusted for age were also statistically quantified. Significance was set at $p \leq 0.05$.

\section{RESULTS}

Although the HIGH group was approximately 5 years younger than the LOW group $(\mathrm{p}<0.01)$, BMI and body composition (\%fat and FFM) were similar between the two groups. Absolute and relative toe grasping maximal strength 
Table 1. Body composition, physical activity, and muscle strength in middle-aged and older women

\begin{tabular}{|c|c|c|c|}
\hline & \multicolumn{2}{|c|}{ Daily step counts (steps/day) } & \multirow[b]{2}{*}{ Overall } \\
\hline & LOW $(<8,000)$ & $\operatorname{HIGH}(\geq 8,000)$ & \\
\hline $\mathrm{N}$ & 28 & 29 & 57 \\
\hline Age, yrs & $69(7)$ & $64(6)^{\dagger}$ & $66.3(6.8)$ \\
\hline Height, $\mathrm{m}$ & $1.53(0.05)$ & $1.53(0.06)$ & $1.53(0.05)$ \\
\hline Body weight, $\mathrm{kg}$ & $52.9(6.1)$ & $51.7(4.5)$ & $52.3(5.4)$ \\
\hline Body mass index, $\mathrm{kg} / \mathrm{m}^{2}$ & $22.7(2.9)$ & $22.1(2.0)$ & $22.4(2.5)$ \\
\hline Body fat, $\%$ & $27.3(5.1)$ & $26.9(5.2)$ & $27.1(5.1)$ \\
\hline Fat-free mass, $\mathrm{kg}$ & $38.3(3.1)$ & $38.2(3.2)$ & $38.2(3.2)$ \\
\hline \multicolumn{4}{|l|}{ Muscle strength } \\
\hline Foot grasping, kg & $11.9(3.1)$ & $14.9(3.3)^{\top}$ & $13.4(3.5)$ \\
\hline Knee extension, $\mathrm{Nm}$ & $104(28)$ & $106(22)$ & $105(25)$ \\
\hline \multicolumn{4}{|l|}{ Relative muscle strength } \\
\hline Foot grasping/Wt, $\mathrm{kg} / \mathrm{kg}$ & $0.23(0.06)$ & $0.29(0.07)^{\llbracket}$ & $0.26(0.07)$ \\
\hline Knee extension/Wt, $\mathrm{Nm} / \mathrm{kg}$ & $1.96(0.48)$ & $2.07(0.45)$ & $2.01(0.46)$ \\
\hline \multicolumn{4}{|l|}{ Physical activity, min/day } \\
\hline Low & $48.3(14.6)$ & $71.4(19.1)^{\natural}$ & $60.1(20.5)$ \\
\hline Moderate & $10.6(7.7)$ & $33.3(13.7)^{9}$ & $22.2(15.9)$ \\
\hline Vigorous & $0.6(0.9)$ & $2.4(1.7)^{\uparrow}$ & $1.54(1.65)$ \\
\hline Low + Moderate & $59.0(17.2)$ & $104.7(18.3)^{\llbracket}$ & $82.2(29.1)$ \\
\hline Moderate + Vigorous & $11.2(8.1)$ & $35.8(14.6)^{\llbracket}$ & $23.7(17.1)$ \\
\hline Step counts, steps/day & $5,456(1,588)$ & $10,406(1,898)^{ף}$ & $7,974(3,041)$ \\
\hline
\end{tabular}

Significant difference from LOW group $\dagger \mathrm{p}<0.01, \uparrow \mathrm{p}<0.001$

Wt: body weight

Table 2. Pearson's and partial (adjusted for age) correlation coefficients between muscle strength and physical activity in middle-aged and older women

\begin{tabular}{lcccc}
\hline & \multicolumn{2}{c}{ Pearson's correlation } & \multicolumn{2}{c}{ Partial correlation } \\
\cline { 2 - 5 } & Grasping & Knee Ex & Grasping & Knee Ex \\
\hline Light + Moderate & $0.415 \dagger$ & $0.306^{*}$ & $0.290^{*}$ & 0.217 \\
Moderate + Vigorous & $0.350 \dagger$ & $0.312^{*}$ & 0.228 & 0.233 \\
Step counts & $0.412 \dagger$ & $0.330^{*}$ & $0.283^{*}$ & 0.242 \\
\hline$* \mathrm{p}<0.05, \dagger \mathrm{p}<0.01$ & & & \\
Grasping, foot grasping strength/body weight; Knee Ex, knee extension strength/body weight
\end{tabular}

were greater $(\mathrm{p}<0.001)$ in the HIGH group than in the LOW group. However, both absolute and relative knee extension strength were similar between the two groups. All 3 intensities (light, moderate, and vigorous) of physical activity were higher in the HIGH group compared with the LOW group (Table 1).

A negative correlation was observed between age and relative toe grasping strength $(\mathrm{r}=-0.480, \mathrm{p}<0.001)$ and between age and relative knee extension strength $(\mathrm{r}=-0.307, \mathrm{p}$ $<0.05)$. Relative toe grasping strength correlated positively with light $(\mathrm{r}=0.320, \mathrm{p}<0.05)$, moderate $(\mathrm{r}=0.345, \mathrm{p}<$ $0.01)$, and vigorous $(\mathrm{r}=0.296, \mathrm{p}<0.05)$ physical activity. Relative knee extension strength correlated positively with moderate $(\mathrm{r}=0.298, \mathrm{p}<0.05)$, and vigorous $(\mathrm{r}=0.358, \mathrm{p}$ $<0.01)$ physical activity, but not with light $(\mathrm{r}=0.203, \mathrm{p}=$ 0.131 ) physical activity. Similarly, both toe grasping and knee extension strength correlated with light plus moderate physical activity as well as moderate plus vigorous physical activity (Table 2). Average step count also correlated with both toe grasping and knee extension strength (Table
2). After adjusting for age, only the duration of light plus moderate physical activity and average step count correlated to toe grasping strength (Table 2).

\section{DISCUSSION}

In the present study, we tested our hypothesis that daily physical activity may contribute to toe grasping strength in middle-aged and older females. The primary findings of this study were that: 1 ) the HIGH group ( $\geq 8,000$ steps/day) had significantly greater toe grasping strength, but not knee extension strength, when compared with the LOW group $(<8,000$ steps/day) strength, 2$)$ toe grasping strength was significantly associated with daily step count, and 3) toe grasping strength significantly correlated with light plus moderate intensity physical activity, but not moderate plus vigorous intensity physical activity.

It has previously been reported that accelerometer (or pedometer)-determined physical activity levels dramatically decrease later in life ${ }^{13-15)}$. In Japanese, for example, average 
yearly step counts were approximately 7,000 steps/day for ages $65-74$ and 5,000 steps/day for ages 75-84 ${ }^{13}$. Kitagawa et al. ${ }^{16)}$ reported a mean value of 8,401 (SD 3404) steps/day in older Japanese females aged 61-87 years (mean age 71 \pm 6 years). Similarly, Yoshioka et al. ${ }^{17)}$ reported an average number of 7,922 steps/day in middle-aged and older adults (females and males) aged 50-69. Physical activity levels of our subjects were similar to the previous studies with an average value of approximately 8,000 steps/day (mean and $\mathrm{SD}, 7,974 \pm 3,041$ ) in middle-aged and older females (mean age $66 \pm 7$ years).

It is generally believed that participation in regular physical activities may be associated with greater maintenance of muscular strength. In the present study, active females ( $\geq 8,000$ steps/day) had $26 \%$ higher toe grasping strength compared with females who were not as physically active $(<$ 8,000 steps/day), while knee extension strength was similar between the two groups. Furthermore, both knee extension and toe grasping strength correlated significantly to average step count, although the correlation coefficient between step count and toe grasping strength was only significant after adjusting for age. Hunter et al. ${ }^{18)}$ investigated the influence of physical activity on muscular strength of knee extensors, plantar flexors, and handgrip in females aged 20 to 89 years. They found that self-reported physically active females were stronger than physically inactive females when absolute strength of the leg muscles was normalized for body weight; however, knee extension strength at ages 60-64, 65-69, and 70-74 were probably similar between the active and inactive females. Leskinen et al. ${ }^{19)}$ reported that active twins had $20 \%$ higher knee extension strength than their inactive co-twins, while the active twins had only $4 \%$ higher mid-thigh muscle cross-sectional area than that of the inactive twins. Our findings in toe grasping strength support the previous studies, whereas the reasons for the difference in knee extension strength between the present and previous studies are not well known. One possible explanation is that most of the previous studies used questionnaires for evaluating the frequency and/or duration of a given type of physical activity during a typical week, and determination of physical activity profile based on questionnaires may or may not be accurate, especially regarding exercise intensity ${ }^{20-22)}$. Thus, this methodological concern may have affected the results reported by the previous studies.

In the present study, toe grasping strength was associated with light plus moderate intensity physical activity. In terms of foot-ground contact time, foot muscles involved in toe grasping may be better activated during light/moderate intensity slow movements than during vigorous intensity fast movements although this may depend on footwear. A previous study examined the effects of low-intensity toe grasp training (which consisted of gathering a towel attached to a weight and passing small bags from one place to another) in older adults and found that postural sway behavior while standing improved following the training ${ }^{3)}$. Although that study did not measure the change in toe grasping strength, it is expected that muscular functions involved in toe grasping may be improved by relatively low-intensity exercise training. Therefore, results from the present and previous studies together suggest that relatively low-intensity exercise would be suitable for improving toe grasping strength.

Another previous study investigated the effect of accelerometer-determined daily physical activity on lower body muscular power in adolescents ${ }^{23}$. The results showed positive correlations between muscular power and vigorous intensity physical activity but not with moderate or light intensity physical activity. In that study, the duration of vigorous intensity physical activity was approximately 14 minutes for female adolescents and 25 minutes for male adolescents. In the present study, the average duration of vigorous intensity physical activity was only 1.5 minutes in middle-aged and older females, and the effect of vigorous intensity exercise on knee extension strength appeared to be limited. Therefore, for improving knee extension strength, the advantage of regular physical activity may depend on the intensity and duration of exercise.

It is possible that older adults with high levels of physical activity in daily life have better balance control compared with less active individuals. Although Aoyagi et al. ${ }^{24)}$ reported no significant association between body sway and level of daily physical activity, many intervention studies have revealed a number of factors that contribute to better balance ${ }^{25)}$. In particular, Gauchard et al. ${ }^{5)}$ reported more improvement in static postural control with Yoga/soft gymnastics (proprioceptive exercise) than with jogging/swimming/cycling (bioenergetic exercise). It has been reported that toe grasping strength is associated with static balance with eyes open as well as with tolerance to falling forward ${ }^{1)}$. Our results, along with those of previous studies, suggest that toe grasping strength may be a contributing factor to the connection between the effects of daily physical activity and balance control.

Several limitations of this study should be mentioned. Because patterns of daily physical activity differ between women and men and our subjects were only women, it is unclear if the results pertain to men. Although the duration of light/moderate physical activity was associated with toe grasping strength, we did not consider the type of exercise performed during daily physical activity. Additional research into these issues is needed.

In summary, active middle-aged and older women had higher toe grasping strength compared with females who were relatively physically inactive. Significant correlations were observed between toe grasping strength and accelerometer- determined physical activity levels, especially the duration of light plus moderate physical activity. These results suggest that toe grasping strength may be associated with the amount of light daily physical activity.

\section{ACKNOWLEDGEMENT}

The authors thank the individuals who participated in this study. This study was partially supported by the Toyo Gakuen University for Aging Research.

\section{REFERENCES}

1) Handa $S$, Horiuchi $K$, Aoki K: A study on the measurement of toes grasping strength and effect of standing postural control. Jpn J Ergon, 2004, 40: 139-147 (in Japanese). [CrossRef] 
2) Abe T, Ogawa M, Thiebaud RS, et al.: Is muscle strength ratio a criterion for diagnosis of site-specific muscle loss? Geriatr Gerontol Int, 2014, 14: 837-844. [Medline] [CrossRef]

3) Kobayashi R, Hosoda M, Minematsu A, et al.: Effects of toe grasp training for the aged on spontaneous postural sway. J Phys Ther Sci, 1999, 11 31-34. [CrossRef]

4) Perrin PP, Gauchard GC, Perrot C, et al.: Effects of physical and sporting activities on balance control in elderly people. Br J Sports Med, 1999, 33: 121-126. [Medline] [CrossRef]

5) Gauchard GC, Gangloff P, Jeandel C, et al.: Influence of regular proprioceptive and bioenergetic physical activities on balance control in elderly women. J Gerontol Med Sci, 2003, 58A: 846-850.

6) Prioli AC, Freitas Júnior PB, Barela JA: Physical activity and postural control in the elderly: coupling between visual information and body sway. Gerontology, 2005, 51: 145-148. [Medline] [CrossRef]

7) Ueno M, Kawai S, Mino T, et al.: [Systematic review of fall-related factors among the house-dwelling elderly in Japan]. Nippon Ronen Igakka Zasshi, 2006, 43: 92-101. [Medline] [CrossRef]

8) Abe T, Kawakami Y, Kondo M, et al.: Prediction equations for body composition of Japanese adults by B-mode ultrasound. Am J Hum Biol, 1994 6: 161-170. [CrossRef]

9) Brozek J, Grande F, Anderson JT, et al.: Densitometric analysis of body composition: revision of some quantitative assumptions. Ann N Y Acad Sci, 1963, 110: 113-140. [Medline] [CrossRef]

10) Soma M, Murata $S$, Kai $Y$, et al.: An examination of limb position for measuring toe-grip strength. J Phys Ther Sci, 2014, 26: 1955-1957. [Medline] [CrossRef]

11) Togo F, Watanabe E, Park H, et al.: How many days of pedometer use predict the annual activity of the elderly reliably? Med Sci Sports Exerc, 2008 40: 1058-1064. [Medline] [CrossRef]

12) Kumahara H, Schutz Y, Ayabe M, et al.: The use of uniaxial accelerometry for the assessment of physical-activity-related energy expenditure: a validation study against whole-body indirect calorimetry. Br J Nutr, 2004, 91: 235-243. [Medline] [CrossRef]

13) Bennett GG, Wolin KY, Puleo E, et al.: Pedometer-determined physical activity among multiethnic low-income housing residents. Med Sci Sports Exerc, 2006, 38: 768-773. [Medline] [CrossRef]

14) Cavanaugh JT, Coleman KL, Gaines JM, et al.: Using step activity moni- toring to characterize ambulatory activity in community-dwelling older adults. J Am Geriatr Soc, 2007, 55: 120-124. [Medline] [CrossRef]

15) Park H, Park S, Shephard RJ, et al.: Yearlong physical activity and sarcopenia in older adults: the Nakanojo Study. Eur J Appl Physiol, 2010, 109: 953-961. [Medline] [CrossRef]

16) Kitagawa J, Omasu F, Nakahara Y: Effect of daily walking steps on ultrasound parameters of the calcaneus in elderly Japanese women. Osteoporos Int, 2003, 14: 219-224. [Medline]

17) Yoshioka M, Ayabe M, Yahiro T, et al.: Long-period accelerometer monitoring shows the role of physical activity in overweight and obesity. Int J Obes, 2005, 29: 502-508. [Medline]

18) Hunter SK, Thompson MW, Adams RD: Relationships among age-associated strength changes and physical activity level, limb dominance, and muscle group in women. J Gerontol A Biol Sci Med Sci, 2000, 55: B264B273. [Medline] [CrossRef]

19) Leskinen T, Sipilä S, Kaprio J, et al.: Physically active vs. inactive lifestyle, muscle properties, and glucose homeostasis in middle-aged and older twins. Age (Dordr), 2013, 35: 1917-1926. [Medline] [CrossRef]

20) Aoyagi Y, Shephard RJ: Steps per day: the road to senior health? Sports Med, 2009, 39: 423-438. [Medline] [CrossRef]

21) Morie M, Reid KF, Miciek R, et al.: Habitual physical activity levels are associated with performance in measures of physical function and mobility in older men. J Am Geriatr Soc, 2010, 58: 1727-1733. [Medline] [CrossRef]

22) Slootmaker SM, Schuit AJ, Chinapaw MJ, et al.: Disagreement in physical activity assessed by accelerometer and self-report in subgroups of age, gender, education and weight status. Int J Behav Nutr Phys Act, 2009, 6: 17. [Medline] [CrossRef]

23) Moliner-Urdiales D, Ortega FB, Vicente-Rodriguez G, et al.: Association of physical activity with muscular strength and fat-free mass in adolescents: the HELENA study. Eur J Appl Physiol, 2010, 109: 1119-1127. [Medline] [CrossRef]

24) Aoyagi Y, Park H, Watanabe E, et al.: Habitual physical activity and physical fitness in older Japanese adults: the Nakanojo Study. Gerontology, 2009, 55: 523-531. [Medline] [CrossRef]

25) Ferreira ML, Sherrington $\mathrm{C}$, Smith $\mathrm{K}$, et al.: Physical activity improves strength, balance and endurance in adults aged $40-65$ years: a systematic review. J Physiother, 2012, 58: 145-156. [Medline] [CrossRef] 